... Jean-Charles Rielle, spécialiste en médecine préventive,

Conseiller national genevois PS, «Monsieur Anti-Tabac»

\title{
«Non, non, je ne suis pas un ascète»
}

\section{Daniel Lüthi}

Texte et photos

danielluethi@gmx.ch
Le 31 mai est son jour car c'est la journée mondiale sans tabac. Son cheval de bataille est en effet la lutte contre les volutes bleues et donc contre l'industrie du tabac. Sa marque de fabrique depuis plus de 20 ans. Sa mission. Il commence à en parler avant même d'en être explicitement sollicité: «la cigarette est une drogue - la nicotine rend plus dépendant que l'héroïne.» On le voit: la dépendance et donc la perte de la liberté individuelle est aussi un sujet d'importance pour ce politicien chevronné.

\section{Une victime chaque heure}

Tout a commencé en 1991, par hasard, à la lecture d'une annonce dans un journal. On cherchait une personne prête à créer un centre de prévention et à le diriger, un centre pour la lutte contre la fumée nocive et contre la consommation de tabac. Jean-Charles Rielle s'en rappelle comme si c'était hier: «L'Organisation mondiale de la santé (OMS) avait déjà désigné le tabagisme comme cause principale évitable de maladies graves et de décès prématurés. En ma qualité de médecin de santé publique, j'ai été directement concerné par cette annonce.» Le CIPRET-Genève (Centre d'Information de l'Association pour la Prévention du Tabagisme) était né; il est devenu son enfant. Il en parle comme un père. Sa réponse se transforme rapidement en un exposé: «Rendez-vous compte, 9000 personnes meurent par année en Suisse des suites du tabagisme, soit une victime chaque heure, et c'est bien plus que le double des accidentés de la route, des

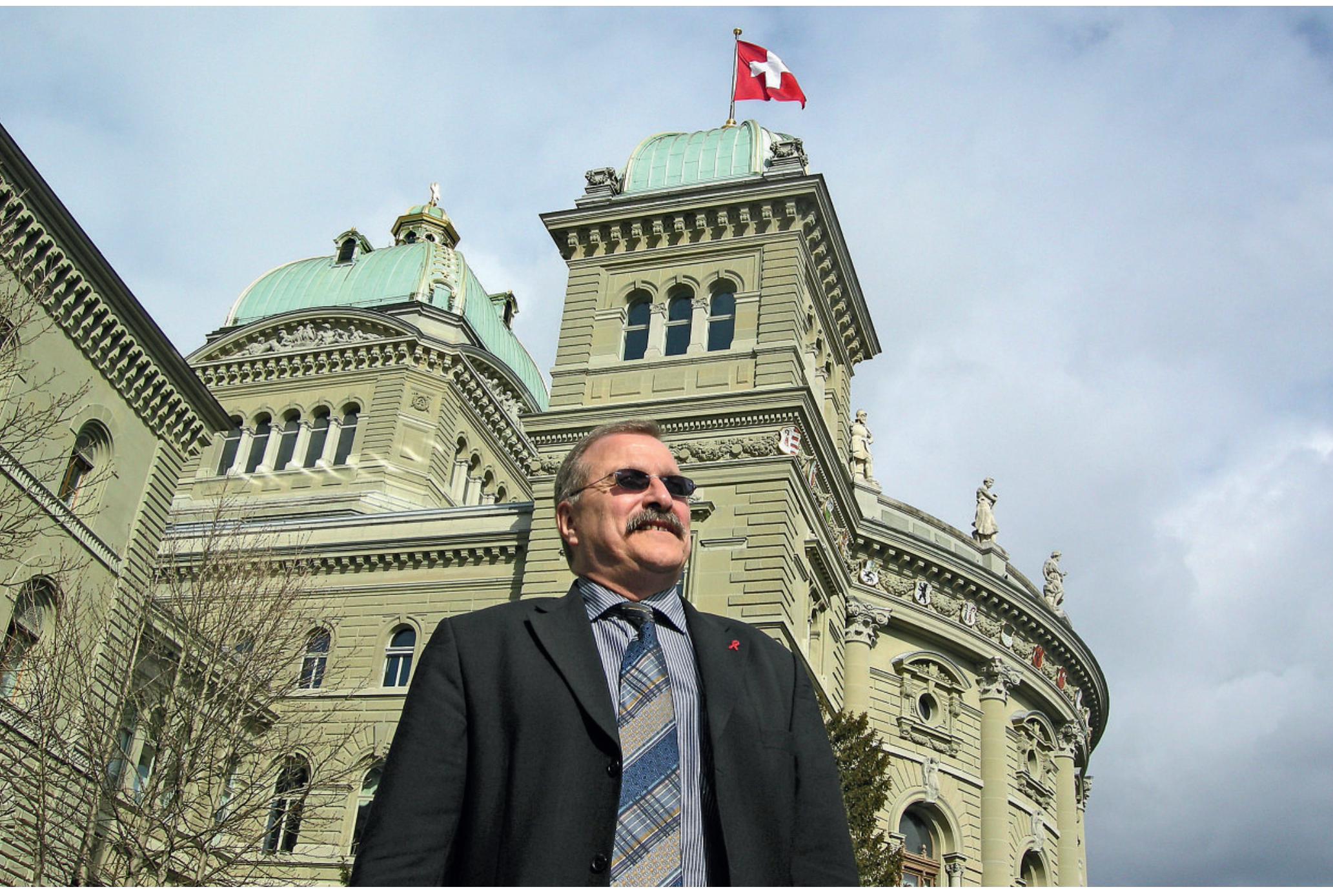


suicides, de l'alcoolisme et d'autres drogues illégales. Je pourrais être cynique et remercier l'industrie du tabac d'aider à remplir les caisses de l'AVS en veillant simultanément à ce qu'un grand nombre de cotisants ne touchent jamais cet argent pour cause de décès prématurés.» Néanmoins, le Dr Rielle ne s'estime pas cynique, il trouve seulement pervers qu'une industrie tue elle-même la moitié de ses clients avec le tabac, si ce produit est utilisé tel qu'il est recommandé par ceux qui le vendent. "Quelle autre industrie agit ainsi, à part celle de l'armement?»

Chaque personne a sa propre histoire de dépendance, le Dr Rielle en est convaincu. Lui-même a commencé à fumer à l'âge de 15 ans à l'internat de St-Maurice pour une raison commune à tous les adolescents, celle de vouloir appartenir au cercle de copains, et il y a mis fin à l'âge de 32 ans, Il a donc fumé pendant 17 ans et considère cet arrêt comme normal: «Il n'y a là aucun mérite mais seulement la conséquence logique de mon activité médicale.» Voir quelqu'un mourir de détresse respiratoire est une expérience qui marque l'esprit.

\section{La solidarité comme attitude de base}

Au revers de ses vestes - blazer et veste de cuir - brille, rouge et bien visible, le ruban du Sida. Un symbole pour la mort de deux personnes que le Dr Rielle connaissait bien et qui sont décédées des suites de l'infection du HIV. Un symbole aussi pour une attitude de base, à savoir la solidarité pour les démunis et les rançonnés du destin. De ce fait, le Dr Rielle est un socialiste convaincu et il fait de la politique au nom du PS. Il ne s'engage pas uniquement pour les travailleurs qui doivent travailler dans des locaux contaminés, c'est-à-dire enfumés, mais par exemple aussi pour les femmes victimes de violence sexuelle.

\section{«Contrairement à l'alcool, il n'existe pas de dose}

\section{acceptable pour le tabac»}

De plus, Jean-Charles Rielle s'engage aussi fortement pour la jeunesse. Il travaille notamment auprès du Tribunal genevois de la jeunesse en qualité de juge assesseur médecin. Là, il s'agit régulièrement d'aider les jeunes à prendre confiance en eux, à les encourager à regarder les difficultés en face, à ne pas les éviter ni à les oublier au moyen de produits rendant dépendant. Dans la vie, il n'est jamais autant demandé à une personne qu'entre l'âge de 12 à 18 ans, dit-il et c'est durant cette phase de transformation que beaucoup font leurs premières expériences avec des drogues. «Mais qui pense déjà après la première gorgée d'une boisson alcoolisée qu'il pourrait faire le premier pas en direction d'une cirrhose du foie?» L'alcool est également un sujet de prédilection pour le Dr Rielle. Son épouse

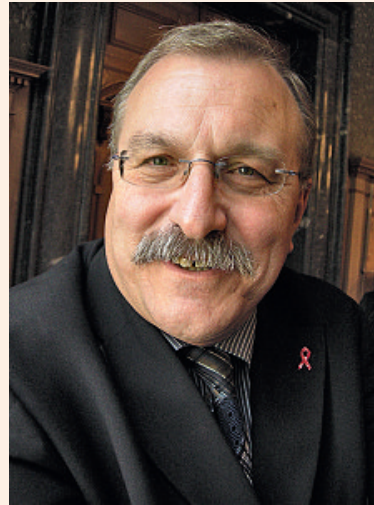

\section{Jean-Charles Rielle}

Né en 1952, le Dr Jean-Charles Rielle a grandi à Sion où ses parents tenaient des commerces de vêtements et un restaurant à Montana. II a étudié la médecine à Genève et s'est intéressé très tôt au domaine de la «Santé publique» (Public Health). Le Dr Rielle est membre du PS Suisse. En 1995, il a été élu au Conseil municipal de Genève et en 2007 au Conseil national où il est notamment membre de la Commission de la sécurité sociale et de la santé publique (CSSS). Il est par ailleurs membre de l'Assemblée parlementaire de la Francophonie.

Pour les élections de l'année prochaine, il s'est porté candidat à la candidature au Conseil administratif de Genève, l'Exécutif de la Ville composé de cinq membres.

En sa qualité de médecin et de politicien, JeanCharles Rielle s'est fait connaître avant tout comme «Monsieur Anti-Tabac», le pape des nonfumeurs. II dirige depuis sa fondation en 1991 le CIPRET à Genève (Centre d'information de I'association pour la prévention du tabagisme). II travaille notamment aussi comme médecin consultant auprès du Service de santé de la jeunesse et est juge assesseur médecin au Tribunal de la jeunesse.

Jean-Charles Rielle est marié pour la cinquième fois. Il a une fille et deux petits-enfants. Ayant acquis par mariage le passeport français en plus de son passeport suisse, il est aussi européen.

Laurence Fehlmann Rielle, ancienne députée et présidente du PS genevois, travaille dans la prévention de l'alcoolisme. Son époux, si décontracté, est-il vraiment une personne hostile aux petits plaisirs de la vie? J.-Ch. Rielle rit de bon cœur. «Certainement pas! Non, non, je ne suis pas un ascète.» Il boit volontiers un verre de vin, quelquefois un de trop. Alors il ne se met plus au volant de sa voiture car il mettrait autrui en 
danger... et nous voici de retour à son cheval de bataille. Au contraire de l'alcool, la consommation de tabac est nocive aussi pour l'entourage, raison pour laquelle il lutte également contre la fumée passive. Il précise «Contrairement à l'alcool, il n'existe pas de dose acceptable pour le tabac.»

Concernant la fumée passive, le $1^{\text {er }}$ mai 2010 est à marquer d'une pierre blanche pour le Dr Rielle et ses compagnons de lutte car, à cette date, le Conseil fédéral a mis en vigueur la nouvelle loi fédérale sur la protection contre le tabagisme passif. Depuis lors, il est interdit de fumer dans tous les lieux publics de Suisse. Dans les restaurants justement, Jean-Charles Rielle est toujours interpellé par sa marque de fabrique «Monsieur Anti-Tabac» et il se voit réduit à cette fonction, ce qui ne l'empêche pas de boire tranquillement une bonne bière avec nous.

\section{«C'était une tromperie qu'il fallait dévoiler»}

\section{L'affaire Rylander}

Le 29 mars 2001 est une autre date clé dans la vie de J.-Ch. Rielle. Avec Pascal Diethelm, fonctionnaire de l'OMS à la retraite, il s'est présenté ce jour-là devant les médias pour dévoiler «un cas de fraude scientifique sans précédent». Les deux hommes avaient en ligne de mire Ragnar Rylander, ancien professeur de santé publique et d'épidémiologie aux universités de Göteborg et de Genève. Ils lui reprochaient d'avoir accepté en cachette, pendant des années, des sommes d'argent de Phillip Morris, le géant de l'industrie du tabac, pour dissimuler les conséquences négatives de la fumée passive par des études scientifiques manipulées. Rylander réagit à cette révélation par une plainte pénale pour diffamation et atteinte à l'honneur contre Diethelm et Rielle. Après un long procès et des sentiments en dents de scie entre succès partiels et déceptions, les deux accusateurs devenus des accusés ont été acquittés.

Le Dr Rielle respire profondément lorsqu'il y pense: «J'ai été complètement occupé par cette affaire pendant trois ans et j'ai même dû être hospitalisé pen- dant 12 jours à cause d'elle. Mais je recommencerais.» Pourquoi? «C'était une tromperie qu'il fallait dévoiler. Il ne s'est pas agi d'un combat contre une personne mais pour un principe.» Chez Jean-Charles Rielle, la lutte pour améliorer la santé de la population est clairement aussi un combat contre l'industrie du tabac.

Une fois de plus, son interlocuteur se demande ce qui motive Jean-Charles Rielle à ce point, ce qui lui donne autant de force pour lutter, car il ne lutte pas seulement à Genève mais aussi au Palais fédéral à Berne. «Alléger les souffrances», c'est la noble tâche du médecin, nous dit-il tranquillement, et aussi «empêcher les souffrances». Aussi celles de toute une population. C'est pourquoi, son objectif est de diminuer constamment le nombre de fumeurs. De $33 \%$, la part de la population s'adonnant au tabagisme a baissé ces dernières années à $27 \%$, constate le Dr Rielle, non sans fierté. Mais il reste au combat, pratiquement jour et nuit: «Je n'ai pas besoin de plus de quatre à cinq heures de sommeil», nous dit cet homme fort occupé et d'un calme olympien, mais qui cherche tout autre chose que la tranquillité: «Le pire que l'on pourrait me faire, serait de me mettre sur une île déserte. J'y mourrais rapidement.»

Et il nous indique aussitôt un autre thème pour lequel il veut agir, le cas échéant au moyen d'une initiative populaire, afin que les personnes qui se font élire à une fonction politique soient tenues de déclarer leurs revenus et de présenter leurs déclarations d'impôts.

\section{«Les gens qui me connaissent me considèrent comme un prototype de I'anti-fanatique»}

Pour effectuer un tel travail de persuasion, faut-il plus que de l'engagement, peut-être même un peu de fanatisme? «En aucun cas», dit Jean-Charles Rielle d'un ton convaincu, «les gens qui me connaissent me considèrent comme un prototype de l'anti-fanatique. Demandez-le à mes amis.»

Dont les fumeurs font aussi partie, comme il le souligne.

\section{La prochaine «rencontre avec...»}

A la fin de chaque mois, le Bulletin des médecins suisses présente une personnalité qui s'engage dans la santé publique. En juin, Daniel Lüthi parlera de sa rencontre avec Barbara Brühwiler, directrice des domaines Soins et Human Resources Management, et membre jusqu'à fin juin de la direction de l'Hôpital universitaire de Zurich. 